\title{
ADAPTACIÓN Y VALIDACIÓN DEL TEST OF EMOTION COMPREHENSION EN ESCOLARES CUBANOS
}

\author{
ADAPTATION AND VALIDATION OF THE \\ TEST OF EMOTION COMPREHENSION IN \\ CUBAN SCHOOLS
}

\author{
LISANDRA ANGULO GALLO ${ }^{1}$, \\ VIVIAN MARGARITA GUERRA MORALES ${ }^{1} \mathrm{Y}$ \\ YIPSANDRA BLANCO CONSUEGRA ${ }^{2}$
}

Cómo referenciar este artículo/How to reference this article:

Angulo Gallo, L., Guierra Morales V. M. y Blanco Consuegra, Y. (2018). Adaptación y Validación del Test of Emotion Comprehension en escolares cubanos [Adaptation and Validation of the Test of Emotion Comprehension in Cuban Schools]. Acción Psicológica, 15(1), 57-70. https://doi.org/10.5944/ap.15.1.21236

\section{Resumen}

La comprensión emocional en los niños incluye todo un conjunto de habilidades para entender las emociones propias y ajenas. Para la evaluación de este constructo fue construido por Pons y colaboradores (Pons, Harris y de
Rosnay, 2004) el Test of Emotion Comprehension (TEC). El trabajo realizado constituye la adaptación de la prueba para la población de niños cubanos de 8 a 10 años, teniendo como objetivo analizar algunas de sus propiedades psicométricas. Para ello se efectuó un estudio cuantitativo, de tipo instrumental, con una muestra de 200 escolares seleccionados aleatoriamente. El procesamiento de los datos se hizo mediante el paquete estadístico SPSS 21.0, especí-

Correspondencia: Lisandra Angulo Gallo. Departamento de Psicología, Facultad de Ciencias Sociales, Universidad Central "Marta Abreu" de Las Villa.

Email: lisandraa@uclv.cu

ORCID: Lisandra Angulo Gallo (https://orcid.org/0000-0002-6533-041X)_y Vivian Margarita Guerra Morales (https://orcid.org/0000-0001-6323-7484)

${ }^{1}$ Universidad Central "Marta Abreu" de Las Villas, Cuba.

${ }^{1}$ Universidad de Camaguey "Ignacio Agramontes".

Recibido: 02 febrero de 2018.

Aceptado: 07 de mayo de 2018. 
ficamente se determinó la confiabilidad de la prueba a partir del método test-retest, así como la correlación entre la escala y el ítem global, empleándose el coeficiente de correlación de Pearson. Se efectuó el análisis factorial confirmatorio, con el uso del programa AMOS 21.0, utilizando modelos de ecuaciones estructurales para determinar la validez de constructo del TEC. Los resultados muestran una elevada correlación entre los resultados de la primera y la segunda aplicación de la prueba que avalan su estabilidad temporal y confiabilidad. Se determinó que el modelo presenta un buen ajuste, según los índices consultados, garantizando su aplicabilidad en los niños cubanos.

Palabras clave: Comprensión emocional; Escolares; Validación; Test.

\section{Abstract}

Emotional understanding in children includes a whole set of skills to understand one's own and others' emotions. For the evaluation of this construct was constructed by Pons and collaborators (Pons, Harris y de Rosnay, 2004) the Test of Emotion Comprehension (TEC). The work carried out constitutes the adaptation of the test for the population of Cuban children aged 8 to 10 years, aiming to analyze some of their psychometric properties. For this, a quantitative study, of an instrumental type, was carried out with a sample of 200 randomly selected students. The data were processed using the statistical package SPSS 21.0. The reliability of the test was determined from the test-retest method, as well as the correlation between the scale and the global item, using the Pearson correlation coefficient. Confirmatory factor analysis was performed using the AMOS 21.0 program, using structural equation models to determine the construct validity of the TEC. The results show a high correlation between the results of the first and the second application of the test that support its temporal stability and reliability. It was determined that the model presents a good fit, according to the indexes consulted, guaranteeing its applicability in Cuban children.

Keywords: Emotional comprehension; School-age childrens; Validation; Test.

\section{Introducción}

La capacidad de discernir y comprender las emociones propias y ajenas es una de las habilidades que compone la competencia emocional, la cual nos permite conocer cómo y por qué las personas actúan como lo hacen, e inferir lo que está ocurriendo emocionalmente a nuestro alrededor (Gómez-Ortiz, 2017). Gracias a la misma es posible alcanzar una interacción más adecuada y ajustada en los contextos sociales, dada la posibilidad de comunicar estados propios y de saber lo que le pasa a los demás, requisitos claves en los procesos de regulación emocional y social (Fernández-Angulo, Quintanilla y Giménez-Dasi, 2016).

Bisquerra et al. (2012) y Casassus (2017) incluyen esta categoría como un eje central de la educación emocional, alegando que ser consciente de las emociones posibilita disfrutar más las agradables y regular las desagradables.

Desde las teorías de la Inteligencia Emocional se ha producido un acercamiento al abordaje de esta y otras competencias. Se reconocen dos tendencias principales; una que enfatiza en la efectividad psicológica y se basa en los llamados modelos mixtos de personalidad y ajuste no cognitivos ligada al logro académico y profesional (BarOn, Franco y Tappata, 2009; Goleman, 2006) y otra que resalta la capacidad cognitiva y se basa en los modelos de inteligencia y desempeño, donde se considera la capacidad para percibir y entender información emocional (Mayer, Salovey, Caruso y Sitarenios, 2003).

Específicamente, la comprensión emocional es abordada desde estas posiciones a partir de variables como conocimiento emocional, conciencia emocional, percepción emocional, donde se incluye la capacidad para reconocer las propias emociones y de otros, discriminar entre emociones reales y fingidas, designar diferentes emociones y reconocer las relaciones entre la palabra y el propio significado de la emoción, para entender las relaciones entre las emociones y situaciones que las suscitan, así como emociones complejas y simultáneas, además de las transiciones entre un estado emocional y otro.

Sin embargo, la autora considera que para su abordaje en la población infantil, se requiere de un mayor nivel de profundización acerca del surgimiento y evolución de la 
comprensión emocional del niño, a su vez, su influencia diferencial en el desarrollo emocional y social de la población infantil y adolescente. También, desde el punto de vista metodológico se destaca la carencia de instrumentos de evaluación que capten mejor la ejecución real de la misma, más allá del uso de los autoinformes, y buscando criterios más claros y objetivos.

Además, el hecho de que esta competencia englobe no solamente la comprensión de las emociones propias sino también las de otras personas conlleva a que se haya relacionado con habilidades de cognición social o de teoría de la mente, lo cual resulta esencial para interpretar la conducta e intenciones comunicativas de las otras personas (Roqueta, Andrés, Clemente y Buils, 2012). Teniendo en cuenta los elementos abordados, se asume el modelo de comprensión emocional propuesto por Harris, Pons y de Rosnay (2004), quienes estudian la comprensión de emociones como una función cognitiva soportada en la interacción social, la cual implica concebir las relaciones sociales y contextuales en las cuales se encuentran inmersos los sujetos, teniendo en cuenta: los actores involucrados y la interacción entre los autores y las normas sociales implicadas en dichas interacciones. Dicha comprensión de las relaciones les permite comprender la conducta propia y ajena, predecir, ocultar, promover y manipular las emociones y por consiguiente las relaciones con los otros (León-Rodríguez y Sierra-Mejía, 2008).

Para Pons et al. (2014) los niños realizan primero una identificación de la situación, lo cual conduce al desencadenamiento de emociones de diferente valencia. Esta primera identificación y, más aún, la conciencia de la emoción suscitada llevan al niño a buscar explicaciones mediante el recuerdo de sucesos análogos o similares. Este desarrollo progresivo de la comprensión emocional lo describen estos autores en nueve niveles de comprensión emocional de acuerdo con la expresión facial, las causas externas, los deseos, las creencias, la influencia de un recuerdo en un estado emocional actual, la posibilidad de controlar un estado emocional, la posibilidad de ocultar una experiencia emocional, emociones contradictorias y emociones sociales-morales.

Bender, Pons, Harris, Esbjom y Reinholdt (2015) encuentran relaciones positivas entre la edad y el nivel gene- ral de comprensión emocional, permitiendo a los autores dividir estos nueve componentes dentro de tres grupos, referidos como fases: la fase externa comprende reconocimiento, recuerdo, y causa; la fase mental comprende los componentes de aspectos mentales de la emoción, es decir, el impacto de los deseos y las creencias, y la distinción entre emociones reales y aparentes; finalmente en la fase reflexiva, la comprensión emocional se caracteriza por la moralidad, regulación, y mezcla. En cuanto a la distinción de emociones reales y aparentes, éstas se encuentran caracterizadas por ser la distinción entre la emoción que se vive y se manifiesta, y la emoción que se experimenta, pero no se demuestra.

A partir de este modelo es posible entender los diferentes niveles de desarrollo por los cuales atraviesa la comprensión emocional infantil, definiéndose la misma como un conjunto de habilidades para comprender las emociones a partir de su expresión externa, el papel que juegan las causas externas, los deseos, las creencias y la memoria en la comprensión de los niños sobre eventos emocionales y los de otros, así como la regulación de una emoción experimentada y la distinción entre la experiencia de dos emociones (Albanese et al., 2006).

Como alternativa metodológica para su evaluación proponen el Test de Comprensión de las Emociones (Test of Emotion Comprehension; TEC). Fue desarrollado con el objetivo de evaluar la capacidad que tienen los niños para comprender las emociones y validado inicialmente con una muestra de 100 niños ingleses de 3,5, 9 y 11 años de edad, igualmente dividido por sexo en cada grupo de edad (Pons, Lawson, Harris y de Rosnay, 2003; Harris y Rosnay, 2004). El TEC ha sido traducido a numerosos idiomas, y estudios en diferentes culturas han corroborado su validez y fiabilidad (Albanese et al., 2006; Fidalgo, 2012; Rocha, Candeias y Roazzi, 2015).

Dada la ausencia de instrumentos que evalúen este constructo en los niños cubanos, en la investigación se realiza su adaptación, teniendo como objetivo analizar algunas de las propiedades psicométricas del TEC. 


\section{Método}

Para el desarrollo del estudio se asumió un paradigma de investigación cuantitativo, desarrollado desde un tipo de estudio instrumental.

\section{Participantes}

La muestra fue seleccionada mediante un muestreo aleatorio estratificado, quedando conformada por $200 \mathrm{ni}-$ ños entre 8 y 10 años, con nivel socioeconómico medio, de diferentes escuelas de la provincia de Villa Clara. Se garantizó la representatividad de cada una de estas edades (63 de 9.67 de 10 y 70 de 10), así como de ambos sexos (99 femenino y 101 masculino). Se obtuvo el consentimiento informado de los padres de todos los niños y se garantizó que no presentasen limitaciones psíquicas e intelectuales que pudiesen obstaculizar la comprensión de las pruebas psicológicas aplicadas, como criterios de inclusión.

\section{Instrumentos de evaluación}

\section{Test de Comprensión Emocional (Test of Emotion Comprehension)}

La prueba fue elaborada por Pons y Harris, 2003, está dividido en diferentes sets de historias en un orden establecido y permite evaluar el nivel general de comprensión de emociones en niños de entre 3 y 12 años de edad. Consiste en un libro de dibujos que presenta en cada página una escena simple. Debajo de cada escena, en la parte inferior de la hoja, se presentan cuatro caras con expresiones faciales diferentes, cada una de las cuales representa un estado emocional distinto. El TEC ha sido traducido a numerosos idiomas, y estudios en diferentes culturas han corroborado su validez y fiabilidad (Albanese y Molina, 2008; Fidalgo, 2012; Roazzi, Dias, Minervino, Roazzi y Pons, 2008; Rocha et al., 2015)

Objetivo: explorar el nivel de comprensión de emociones en niños en edad escolar.

Materiales: libro del TEC, protocolo de respuestas y lápiz.
Procedimiento: el procedimiento se divide en dos pasos: (1) El examinador le muestra al niño una escena en donde la cara del personaje está en blanco y le lee la historia que plantea una situación. (2) Después de escuchar la historia, se le pide al niño que le haga una atribución emocional al personaje principal señalando el más apropiado de los 4 estados emocionales posibles (las respuestas del niño son no verbales).

Calificación: el test se divide en 9 bloques presentados en un orden preestablecido. Cada bloque evalúa un componente particular de la comprensión de emociones:

1. Reconocimiento de las emociones en base a la expresión facial.

2. Comprensión de las causas externas de las emociones.

3. Comprensión de la influencia de un recuerdo en un estado emocional presente.

4. Comprensión de las emociones basadas en deseos.

5. Comprensión de las emociones basadas en creencias.

6. Comprensión de la posibilidad de esconder un estado emocional

7. Comprensión de la posibilidad de controlar una emoción experimentada.

8. Comprensión de emociones mixtas.

9. Comprensión de emociones morales.

A su vez, estos componentes se agrupan en tres niveles de la comprensión emocional, organizados jerárquicamente: nivel externo (componentes 1-3), nivel mental (componentes 4-6) y nivel reflexivo (7-9).

Se asigna un punto a cada componente al cual el niño responde correctamente. El niño puede obtener un nivel de comprensión de emociones con un máximo de 9 puntos y un mínimo de 0 puntos.

\section{Procedimiento}

La adaptación fue realizada considerando los pasos propuestos por la Comisión Internacional de Test (Mikulic y Crespi, 2009) ). Primeramente, se obtuvieron los permi- 
sos legales para la adaptación y empleo de la técnica por parte de uno de los autores: Francisco Pons. Luego fue traducida al español por dos traductores de habla inglesa y valorada por cinco especialistas cubanos (tres en Filología y dos en Lengua Inglesa), con categorías científicas de doctor, con más de 20 años de experiencia profesional, efectuándose las correcciones que permitieron garantizar su ajuste cultural y gramatical. Los cambios realizados fueron en los términos siguientes: enfadado por bravo, ómnibus por guagua, canicas por bolas y Coca cola por refresco. Con posterioridad se sometió la prueba a un pilotaje de 30 sujetos para su adecuación semántica a las características culturales del contexto y verificar la comprensión de los ítems y de las instrucciones. Los mismos presentaron en su composición características similares a los participantes de la muestra definitiva. Como resultado del pilotaje, se cambió el procedimiento de puntuación en el componente IV (creencia) con el fin de que los resultados sean más similares a los originales, al igual que en la adaptación italiana (Rocha et al., 2015), se consideraron correctas ambas respuestas: felices y bien. Esta decisión se ha tomado porque aproximadamente el $40 \%$ de todos los niños ofreció como respuesta la alternativa "bien", los especialistas reconocieron que es plausible debido a que, en términos de contenido, en español la palabra para $\mathrm{OK}$ ("bien") puede ser utilizado como sinónimo de feliz (es posible que el conejo se sienta bien).

Una vez realizadas las correcciones pertinentes, se administró la técnica a los 200 participantes que integraron la muestra, a los cuales se informaron los objetivos del estudio y se obtuvo el consentimiento informado de sus padres. La administración de TEC tomó alrededor de 15 a 20 minutos con cada niño. Se aplicó en horario matutino, garantizando adecuadas condiciones de iluminación, privacidad y que no existiesen distractores que pudiesen interferir en los resultados. Transcurrido un mes, fue aplicado el TEC nuevamente a los mismos niños para contrastar la estabilidad de las respuestas, como indicador de confiabilidad. No se constataron valores perdidos ni mortalidad muestral.

\section{Análisis de datos}

El procesamiento de los datos se efectuó mediante el paquete estadístico SPSS 21.0 y el programa AMOS 21.0, utilizando modelos de ecuaciones estructurales.

Se calcularon los estadísticos descriptivos de la muestra y se analizaron las propiedades psicométricas del test, específicamente la confiabilidad a partir del método testretest y la correlación entre la escala y el ítem global, respectivamente, empleándose en coeficiente de correlación de Pearson.

Para evaluar la validez de constructo, constatando el ajuste cultural del modelo de comprensión emocional de Harri y Pons para la población de niños cubanos se empleó el Análisis Factorial Confirmatorio (AFC). Se evalúa el ajuste del modelo comprobando los estadísticos de bondad de ajuste que la mayoría de los autores defienden y que siguiendo a Byrne (2001) son: la prueba $\chi 2$ y la razón $\chi 2$ /grados de libertad, se utiliza el de bondad de ajuste comparativo (CFI), el índice de bondad de ajuste general (GFI) y el índice de bondad de ajuste corregida (AGFI), el índice de Tuker-Lewis (TLI), la raíz del residuo cuadrático promedio de aproximación (RMSEA) (Pérez, Chacón y Moreno, 2000).

\section{Resultados}

La representación gráfica (Figura 1) es el modelo que evalúa el ajuste del modelo a los datos observados. Los cuadrados representan las variables observables (componentes de la CE evaluados en el test). Las variables no observables o latentes se presentan como óvalos, es decir, las dimensiones del test. Los círculos, en frente de los ítems, representa el error que es la influencia de fuentes de variabilidad únicas asociadas a las variables observables. Las flechas unidireccionales que salen de las variables latentes a las observables representan un efecto causal de la variable latente sobre la observable. Las flechas curvas bidireccionales entre las variables latentes indican covarianza entre ellas. 


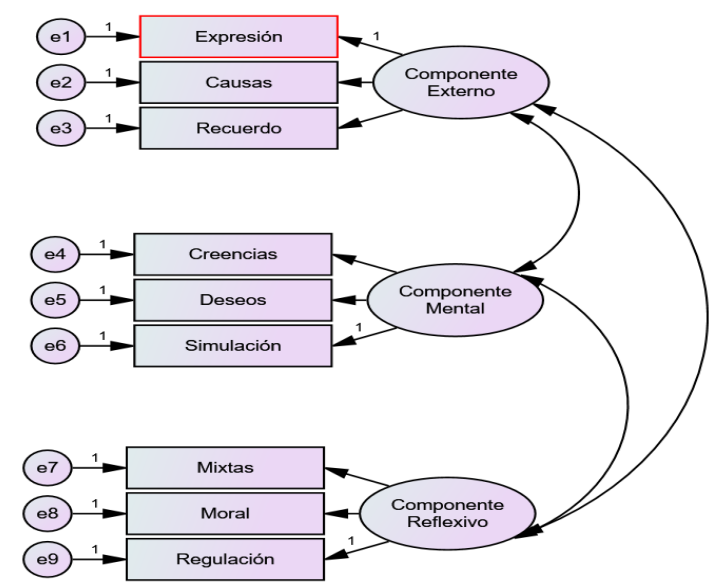

Figura 1. Análisis Factorial Confirmatorio, utilizando Modelo de Ecuaciones Estructurales.

$\chi^{2}=28.566(\mathrm{~g} . \mathrm{I}=24), p=.237, \chi^{2} / \mathrm{g} . \mathrm{I}=1.190 ; \mathrm{GFI}=.969, \mathrm{AGFI}=.943, \mathrm{CFI}=.909, \mathrm{TLI}=.863, \mathrm{RMSEA}=.031$.

Se consideró que el modelo presenta un buen ajuste, de acuerdo con los datos que se muestran en la Figura 1, pues $\chi^{2}$ fue no significativo y $\chi^{2} /$ grados de libertad presentó valores inferiores a 2 , indicando un adecuado ajuste general del modelo, además, todos los índices estuvieron dentro de los valores aceptados: NFI, GFI y TLI presentó valores cercanos a 1 . Con respecto al RMSEA, los valores fueron inferiores a 0.06 .

En la Tabla 1 se puede apreciar la existencia de correlaciones directas, altamente significativas entre cada uno de los componentes que evalúa el test y el constructo comprensión emocional (correlación ítem-total), aunque no se

Tabla 1 distinguen correlaciones entre ellos. Estos también se encontraron correlacionados con los factores o niveles en los cuales se agrupan: Externo (Expresión $r=.359$; Causas $r=.401$; Recuerdo $r=.810$ ), Mental (Creencias $r=.635$; Deseos $r=.562$; Control de la expresión emocional $r=.598$ ) y Reflexivo (Regulación $r=.649$; Emociones mixtas $r=.657$; Emociones morales $r=.379$ ).

A la aplicación del re-test, los datos revelaron que la correlación entre ambas mediciones de la $\mathrm{CE}$, transcurrido un mes, fue fuerte en extremo, directa y altamente significativa $[\mathrm{r}(200)=.889, p<.001]$ con puntuaciones medias muy cercanas: $\mathrm{CE}$ Pre $=7.40$ y CE Post $=7.48$. También

\section{Análisis correlacional ítems- total}

\begin{tabular}{lrrrrrrrrrr}
\hline Variables & \multirow{2}{*}{ Expresión } & Causas & Recuerdo & Creencias & Deseos & $\begin{array}{c}\text { Control } \\
\text { EE }\end{array}$ & Regulación & Morales E. Mixtas & CE \\
\hline Expresión & 1 & .033 & .078 & .102 & -.025 & .055 & $.219^{* *}$ & .049 & .108 & $.223^{* *}$ \\
Causas & .033 & 1 & .011 & .026 & $.209^{* *}$ & $.305^{* *}$ & $.252^{* *}$ & .061 & -.055 & $.377^{* *}$ \\
Recuerdo & .078 & .011 & 1 & .050 & -.095 & .040 & -.090 & .009 & .000 & $.185^{* *}$ \\
Creencias & .102 & .026 & .050 & 1 & -.046 & .022 & .064 & -.080 & .042 & $.344^{* *}$ \\
Deseos & .025 & $.209^{* *}$ & -.095 & .046 & 1 & .139 & .091 & $.195^{* *}$ & -.057 & $.407^{* *}$ \\
Control.EE & .055 & $.305^{* *}$ & -.040 & .022 & .139 & 1 & $.186^{* *}$ & .069 & $.147^{*}$ & $.550^{* *}$ \\
Regulación & $.219^{* *}$ & $.252^{* *}$ & .090 & .064 & .091 & $.186^{* *}$ & 1 & .025 & .045 & $.481^{* *}$ \\
Morales & .049 & .061 & .009 & .080 & $195^{* *}$ & .069 & .025 & 1 & -.027 & $.286^{* *}$ \\
E. Mixtas & .108 & .055 & .000 & .042 & -.057 & $.147^{*}$ & .045 & -.027 & 1 & $.450^{* *}$ \\
\hline
\end{tabular}

Nota. ${ }^{*} p<.05,{ }^{* *} p<.001$. EE: Expresión Emocional, E. Mixtas: Emociones Mixtas, CE: Comprensión Emocional. 
fueron fuertes y significativas las correlaciones establecidas para cada uno de los niveles y componentes del TEC, tal como se muestra en la Tabla 2.

Tabla 2

Análisis correlacional de las dimensiones del TEC, teniendo en cuenta los resultados de la primera y la segunda aplicación

\begin{tabular}{lc}
\hline Variables & $\begin{array}{r}\text { Correlación de Pearson } \\
\text { Sig. (bilateral) }\end{array}$ \\
\hline Expresión & $.939^{* *}$ \\
& .000 \\
Causas & $.911^{* *}$ \\
Recuerdo & .000 \\
& $.785^{* *}$ \\
Creencias & .009 \\
& $.859^{* *}$ \\
Deseos & .000 \\
& $.810^{* *}$ \\
Control.EE & .000 \\
Regulación & $.825^{* *}$ \\
& .000 \\
Moral & $769^{* *}$ \\
E. Mixtas & .000 \\
\hline Nota. ${ }^{*} p<.05,{ }^{* *} p<.001$. & $.823^{* *}$ \\
\end{tabular}

En la Tabla 3 se pueden apreciar los resultados promedios y la desviación típica para cada uno de los componentes de la comprensión emocional representados en el modelo cuyo ajuste fue evaluado, así como las puntuaciones en bruto que obtuvieron los niños en la calificación por niveles y de manera general, destacándose una tendencia descendente. En el ítem correspondiente a creencias se ilustran los valores atendiendo a los dos tipos de respuesta aceptados, tal como se explicó en el procedimiento. De manera general se aprecia una tendencia descendente, siendo los indicadores que se agrupan en el componente mental y reflexivo los de mayor complejidad.

Al realizar una comparación de estos resultados según el sexo y la edad se encontró que los niños mayores mos- traron mejor comprensión de emociones mixtas $\left(\chi^{2}=9.512, p=0.009\right)$ y las niñas alcanzaron mejores puntuaciones en la dimensión reflexiva $\left(\chi^{2}=9.761\right.$, $p=0.021)$. En el resto de los indicadores y componentes no se hallaron diferencias significativas.

\section{Tabla 3}

Estadísticos descriptivos de los resultados obtenidos por los niños en el TEC

\begin{tabular}{lcll}
\hline Componentes & Valores & Media & DT \\
\hline Expresión & $0-1$ & 0.97 & 0.184 \\
Causas & $0-1$ & 0.97 & 0.171 \\
Recuerdo & $0-1$ & 0.86 & 0.353 \\
Creencias & $0-1$ & $0.60(0.85)^{*}$ & 0.492 \\
Deseos & $0-1$ & 0.81 & 0.397 \\
Control de la EE & $0-1$ & 0.83 & 0.381 \\
Regulación & $0-1$ & 0.77 & 0.422 \\
Emociones Mixtas & $0-1$ & 0.69 & 0.464 \\
Moral & $0-1$ & 0.93 & 0.264 \\
N. Externo & $0-3$ & 2.79 & 0.420 \\
N. Mental & $0-3$ & 2.39 & 0.692 \\
N. Reflexivo & $0-3$ & 2.23 & 0.760 \\
Comprensión Emocional & $3-9$ & 7.40 & 1.199 \\
\hline Nota. "Variable recodificada. & & &
\end{tabular}

Nota. *Variable recodificada.

\section{Discusión}

La investigación se realizó con el objetivo de analizar las propiedades psicométricas del TEC, con vistas a su empleo para la evaluación de la comprensión emocional en escolares cubanos. Los datos de este estudio muestran que el modelo de la escala completa se ajusta bien a los datos, esto también se observa en cada uno de los dominios, conservándose la estructura factorial de 9 componentes agrupados en tres niveles propuesta por los autores originales del instrumento. Ello evidencia su ajuste cultural, lo cual ofrece garantías para su aplicación. La equivalencia transcultural de la prueba también ha sido demostrada en otros procesos de adaptación, por ejemplo, a lengua portuguesa (Rocha, Roazzi, Lopes da Silva, Candeias, Minervino, 2015) e italiana (Albanese y Molina, 2008), destacándose la validez de la propuesta teórica-metodológica para la evaluación del constructo comprensión emocional. Los resultados de la prueba también son de elevada confiabilidad, pudiéndose distinguir una alta estabilidad temporal durante su aplicación pasado un mes, los resultados de las 
correlaciones fueron más elevados que en los procesos de validación mencionados con anterioridad, pues en otros el tiempo transcurrido previo al retest ha sido mayor.

Las funciones de información de los ítems se acomodan al patrón esperado teóricamente, con una estructuración jerárquica que determina que los últimos ítems deben medir con más precisión los niveles altos del rasgo, pues son los de resultados más bajos, así como los de mayor poder discriminativo, lo cual se pudo apreciar en las correlaciones ítems-total. Estos resultados son similares a los de otros estudios realizados para la validación de esta prueba en otras poblaciones (Albanese y Molina, 2008; Fidalgo, Pons, Quintanilla, 2012; Roazzi, Dias, Minervino y Pons, 2008), ello pudiese explicar en alguna medida la no necesaria correlación entre los indicadores encontrada en este estudio.

No se presenciaron diferencias significativas de acuerdo al sexo y la edad, exceptuando algunos aspectos del nivel reflexivo como el reconocimiento de emociones mixtas o ambivalencia emocional, lo cual contrasta con planteamientos de algunos autores (Pons et al., 2014), ello se debe a que el rango de edades era muy pequeño, lo cual sugiere que debe ampliarse la investigación, aplicándose en otras edades dentro de la población infantil.

En conclusión, este estudio permite verificar la adaptación del instrumento y la factibilidad de su aplicación desde una primera aproximación. Sin embargo, se recomienda continuar avanzando en el hallazgo de evidencias de otros tipos de validez a partir de otras medidas psicométricas en futuros estudios con muestras más grandes y estratificadas. Ello permitiría la realización de análisis de validación cruzada para examinar si el mismo modelo factorial y los valores de los parámetros se mantienen a través del sexo, edad, así como otras variables. El empleo de la técnica en la población cubana tendrá un impacto significativo en el campo de la evaluación y diagnóstico infantil, donde sería interesante estudiar, no sólo las particularidades de del desarrollo afectivo en muestras sanas, sino que favorecería la detección temprana e intervención en desviaciones en este sentido, en diferentes muestras clínicas.

\section{Referencias}

Albanese, O., Grazzani, I., Molina, P., Antoniotti, C., Arati, L., Farina, E. y Pons, P. (2006). Children's Emotion Understanding: Preliminary Data of the Italian Validation Project of Test of Emotion Comprehension (T.E.C.) En F. Pons, M.F. Daniel, L.Lafortune, P.A. Doudin, O. Albanese (Ed.), Toward Emotional Competences (pp.39-53) Aalborg (Denmark): Aalborg University Press.

Albanese, O. y Molina, P. (2008). Lo sviluppo della comprensione delle emozioni e la sua valutazione. $\mathrm{La}$ standardizzazione italiana del Test di Comprensione delle Emozioni (TEC) [The sviluppo della comprensione delle emozioni e la sua valutazione. The Italian standardization of the Emotion Comprehension Test (TEC)]. Milano, Italia: Unicopli.

Bar-On, R., Franco, M. y Tappata, L. (2009). EQ-I: emotional quotient inventory: manuale. USA: Giunti OS.

Bender, P., Pons, F., Harris, P., Esbjom, B. y Reinholdt, M. (2015). Emotion Underestanding in Clinically Anxious Children: A Preliminary Investigation. Frontiers in Psychology, 6, 1916 https://doi.org/10.3389/fpsyg.2015.01916

Bisquerra, R., Punset, E., Mora, F., García Navarro, E., É, L.-C., Pérez-González, J. C. y Planells, O. (2012). ¿Cómo educar las emociones? La inteligencia emocional en la infancia y la adolescencia [How to educate the emotions? Emotional intelligence in childhood and adolescence]. Esplugues de Llobregat, España: Hospital Sant Joan de Déu.

Byrne, B. (2001). Multivariate Applications Book series. Structural Equation Modeling with AMOS: Basic Concepts, Applications, and Programming. Mahwah, NJ: Lawrence Erlbaum Associates. https://doi.org/10.1207/S15327574IJT0101_4. 
Casassus, J. (2017). Una introducción a la educación emocional [An Introduction to Emotional Education]. Revista Latinoamericana de Políticas y Administración de la Educación 7, 121-130. Recuperado

de http://www.revistasuntref.com.ar/index.php/relapa e/article/view/84/71

Fernández-Angulo, A., Quintanilla, L. y Giménez-Dasi, M. (2016). Dialogando sobre emociones con niños en riesgo de exclusión social: un estudio preliminar [Talking about Emotions with Children at Risk of Social Exclusion: A preliminary Study]. Acción Psicológica, 13(1), 191-206. https://doi.org/10.5944/ap.13.1.15787

Fidalgo, A. M., Pons, F. y Quintanilla, L. (2012). Análisis exploratorio de las propiedades psicométricas y del DIF en el Test de Comprensión de las Emociones [Exploratory Analysis of the Psychometric and DIF Properties in the Emotion Comprehension Test]. Comunicación presentada al VI Congreso Internacional y XI Nacional de Psicología Clínica, Santiago de Compostela, España.

Goleman, D. (2006). Emotional Intelligence. USA: Bantam.

Gómez-Ortiz, O., Romera, EM. y Ortega-Ruiz, R. (2017). La competencia para gestionar las emociones y la vida social y su relación con el fenómeno del acoso y la convivencia escolar [The Skill to Manage Emotions and Social Life and their Link to Bullying and Good Relationships at School]. Revista Interuniversitaria de Formación del Profesorado, 88 (31.1), 27-39. Recuperado de https://dialnet.unirioja.es/servlet/articulo?codigo= 5980921

León-Rodríguez, D. A. y Sierra-Mejía, H. (2008). Desarrollo de la comprensión de las consecuencias de las memociones [Development of Understanding of the Consequences of Memories]. Revista Latinoamericana de Psicología, 40(1), 35 45. https://doi.org/10.14349/rlp.v40i1.354
Mayer, J. D., Salovey, P., Caruso, D. R. y Sitarenios, G. (2003). Measuring Emotional Intelligence with the MSCEIT V2. 0. Emotion, 3(1), 97.

Mikulic, IM. y Crespi, M. (2009). Lineamientos para la adaptación transcultural de instrumentos de evaluación psicológica de la Comisión Internacional de Test (ITC): su aplicación en el Inventario de Personalidad de California [International Test Commission (ITC) Guidelines on Crosscultural Test Adaptation in Psychological Assessment: California Personality Inventory Adapted Test]. I Congreso Internacional de Investigación y Práctica Profesional en Psicología. XVI Jornadas de Investigación Quinto Encuentro de Investigadores en Psicología del MERCOSUR. Facultad de Psicología Universidad de Buenos Aires, Buenos Aires.

Pérez, J., Chacón, S. y Moreno, R. (2000). Validez de constructo: el uso de análisis factorial exploratorioconfirmatorio para obtener evidencias de validez [Construct Validity: The Use of Factor Analysis]. Psicothema., 12, 442-446. Recuperado de http://www.psicothema.es/pdf/601.pdf

Pons, F., Harris, P. L. y de Rosnay, M. (2004). Emotion Comprehension between 3 and 11 years: Developmental Periods and Hierarchical Organization. European Journal of Developmental Psychology, 1(2), 127-152. https://doi.org/10.1080/17405620344000022

Pons, F., Lawson, J., Harris, P. L. y de Rosnay, M. (2003). Individual Differences in Children's Emotion Understanding: Effects of Age and Language. Scandinavian Journal of Psychology, 44(4), 347-353.

Pons, F., Rosnay, M., Bender, P., Doudin, A., Harris, P. y Giménez-Dasí, M. (2014). The Impact of Abuse and Learning Difficulties on Emotion Understanding in Late Childhood and Early Adolescence. The Journal of Genetic Psychology: Research and Theory on Human Development, 
175(4),

301-17.

https://doi.org/10.1080/00221325.2014.903224

Roazzi, A., Dias, M., Minervino, C., Roazzi, M. y Pons, F. (2008). Compreensão das emoções em crianças: Estudo transcultural sobre a validação do Teste de Compreensão da Emoção TEC (Test of Emotion Comprehension) [Understanding Emotions in Children: A Cross-Cultural Study on the Validation of the Emotion Comprehension Test (TEC)]. Paper presented at the Actas da XIII Conferencia Internacional de Avaliação Psicológica.

Rocha, A., Roazzi, A. , Lopes da Silva, A., Candeias, A., Minervino, A., y Roazzi, M. y. Pons F. (2015). Test of Emotion Comprehension: Exploring the underlying Structure through Confimatory Factor
Analysis and Similarity Structure Analysis. En A. Roazzi (Ed.), Facet Theory: Searching of estructure in complex social, cultural an psychological phenomena (pp. 66-85). Recife: Editora UFPE. Recuperado de https://dspace.uevora.pt/rdpc/handle/10174/10838

Roqueta, R., Andrés, C., Clemente, E. y Buils, F. (2012). Cognición Social y competencia pragmática. El caso de los niños y niñas con Trastorno Específico del Lenguaje [Social Cognition and Pragmatic Competence. The Case of Children with Specific Language Impairment]. International Journal of Psychological Research, 5, 59-69. Recuperado de http://www.redalyc.org/articulo.oa?id=299023539 008

\title{
ADAPTATION AND VALIDATION OF THE TEST OF EMOTION COMPREHENSION IN CUBAN SCHOOLS
}

\author{
LISANDRA ANGULO GALLO ${ }^{1}$, \\ VIVIAN MARGARITA GUERRA MORALES ${ }^{1} \mathrm{Y}$ \\ YIPSANDRA BLANCO CONSUEGRA ${ }^{2}$
}

\section{EXTENDED SUMMARY}

\section{Introduction}

The ability to identify and understand emotions of ourselves or others is one of the skills that make up emotional competence, which allows us to know how and why people act as they do, and to infer what is happening emotionally around us (Gómez-Ortiz, 2017). Emotion understanding facilitate a more appropriate and adjusted interaction in social contexts, given the possibility of communicating one's own states and knowing what happens to others, it is key requirements in the processes of emotional and social regulation (Fernández-Angulo, Quintanilla and GiménezDasi, 2016).

Bisquerra et al. (2012) and Casassus (2017) include this category as a central aspect of emotional education, they consider that makes it possible to enjoy more pleasant emotions and regulate unpleasant. This this theoretical construct has been studied from different theoretical positions (Bar-On, Franco and Tappata, 2009; Goleman, 2006; Mayer, Salovey, Caruso and Sitarenios, 2003). 
However, the authors think that, for its approach in the child population, it requires a greater level of deepening about the formation and development of the emotional understanding of the child. Also, from a methodological aspect, there are few measures to evaluation that better capture the real execution of it, beyond the use of self-reports, and looking for clearer and more objective criteria (Roqueta, Andrés, Clemente y Buils, 2012). Therefore, in this research, it is assumed the model of emotional comprehension proposed by Harris, Pons and de Rosnay (2004), who study the understanding of emotions as a cognitive function supported in social interaction, which implies conceiving the social and contextual relationships in which the subjects are immersed, taking into account: the actors involved and the interaction between the authors and social norms involved in these interactions (León-Rodríguez and Sierra-Mejía, 2008).

For Pons et al. (2014), children perform first an identification of the situation, leading to the outbreak of emotions of different valence. This first identification and awareness of emotion take the child to seek explanations by the memory of analogous or similar events. This progressive development of emotional understanding is described by these authors in nine levels of emotional understanding grouped in three aspect: external, mental and reflective. These components are: recognition of facial expression, external causes, desires, beliefs, the influence of a remember on a current emotional state, the ability to control an emotional state, the possibility of hiding an emotional experience, contradictory emotions and socialmoral emotions (Albanese et al., 2006).

As a methodological alternative for their evaluation they propose the Test of Emotion Comprehension (TEC). It was developed with the aim to evaluate the ability of children to understand emotions and it was validated initially with a sample of 100 English children of 3.5, 9, and 11 years of age, equally divided by sex in each age group (Pons, Lawson, Harris and de Rosnay, 2003; Harris and Rosnay, 2004). The TEC has been translated into numerous languages, and studies in different cultures have corroborated its validity and reliability (Albanese et al., 2006; Fidalgo, 2012; Rocha, Candeias and Roazzi, 2015).
At present, it is required instruments to assess this category in Cuban children, that is why, this research carried out TEC adaptation, aiming to analyze some of its psychometric properties.

\section{Method}

The research is based is a quantitative paradigm, there were used a type of instrumental study.

\section{Participantes}

The sample was selected through a stratified random sampling, integrated for 200 children between 8 and 10 years old, with a medium socioeconomic level, from different schools of Villa Clara province. It was guaranteed representativeness of each of these ages (63 of 9.67 of 10 and 70 of 10), as well as of both sexes (99 female and 101 male).

\section{Procedure}

The adaptation was made considering the steps proposed by the International Test Commission (Mikulic and Crespi, 2009). First, legal permits were obtained for the adaptation and use of the technique by one of the authors: Francisco Pons. Then it was translated into Spanish by two English-speaking translators and valued by five Cuban specialists (three in Philology and two in English), with scientific categories of science doctor, with more than 20 years of professional experience, making the corrections that allowed to guarantee their cultural and grammatical adjustment. The changes made were in the terms following: angry for bravo, omnibus for bus, marbles for balls and Coke for refreshment.

Later, the test underwent a pilot with 30 children for study its semantics adequacy and to the cultural context, as well as verify understanding of items and instructions adequacy. Once the pertinent corrections were made, the technique was administered to the 200 participants who integrated the sample. It was informed the objectives of 
the study to them and was obtained the informed consent of their parents. The administration of TEC took around 15 to 20 minutes with each child. After one month, the TEC was applied again to the same children to test the stability of the answers, as an indicator of reliability.

\section{Date Analysis}

The data was processed using the statistical package SPSS 21.0 and the AMOS 21.0 program. The descriptive statistics of the sample were calculated and the psychometric properties of the test were analyzed, specifically the reliability based on the test- retest method and the correlation between the scale and the global item, respectively, using the Pearson correlation coefficient. The Confirmatory Factor Analysis (CFA) was used to assess the construct validity, confirming the cultural adjustment of the Harri and Pons emotional comprehension model for the population of Cuban children.

\section{Results}

\section{Confirmatory Factor Analysis}

It was considered that the model presents a good fit, because $\chi^{2}$ was not significant $\left(\chi^{2}=28.566\right.$, g. $1=24$, $p=.237)$ and $\chi^{2} /$ degrees of freedom presented values lower than $2\left(\chi^{2} / \mathrm{g} .1 .=1.190\right)$, indicating an adequate general adjustment of the model. In addition, all the indices were within the accepted values: NFI, GFI and TLI presented values close to $1(\mathrm{GFI}=.969, \mathrm{AGFI}=.943$, $\mathrm{CFI}=.909, \mathrm{TLI}=.863)$. With respect to the RMSEA, the values were lower than $0.06(\mathrm{RMSEA}=.031)$.

\section{Correlation Analysis}

It was appreciated the existence of highly significant correlations between each of the test components and the emotional understanding construct (item-total correlation), although no correlations between them are distinguished. Also, these were found correlated with the factors or levels in which they are grouped: External (Expression $r=.359$, Causes $r=.401$, Rememory $r=.810$ ), Mental
(Beliefs $r=.635$, Desires $r=.562$ control of the emotional expression $r=.598$ ) and Reflective (Regulation $r=.649$, Mixed emotions $r=.657$, Moral emotions $r=.379$ ).

After the re-test has been applied, the data revealed that the correlation between both EU measurements, after one month, was extremely strong, direct and highly significant $[\mathrm{r}(200)=.889, p<.001]$ with average scores very close to each other: $\mathrm{CE}$ Pre $=7.40$ and CE Post $=7.48$. Also, the correlations established for each of the TEC levels and components were strong and significant.

\section{Descriptive Analysis of the variables}

The average results and standard deviation for each of the components of emotional understanding represented in the model whose adjustment was evaluated, and the raw scores obtained by the children, were showed a downward trend, being the indicators that are grouped in the mental and reflective component those of greater complexity.

When comparing these results according to sex and age, it was found that older children showed better understanding of mixed emotions $\left(\chi^{2}=9.512, p=0.009\right.$ and girls obtained higher scores in the reflective dimension $\left(\chi^{2}=9.761, p=0.021\right)$. In the rest of the indicators and components, no significant differences were found.

\section{Discussion}

The data from this study show that the model of the full-scale fits well with the data, this is also observed in each of the domains, keeping the factorial structure of 9 components grouped into three levels proposed by the original authors of the instrument. This results demostrate its cultural adjustment, which offers guarantees for its application. The transcultural equivalence of the test has also been demonstrated in other adaptation processes, for example, to the Portuguese language (Rocha, Roazzi, Lopes da Silva, Candeias, Minervino, 2015) and Italian (Albanese and Molina, 2008), highlighting the validity of the theoretical-methodological proposal for the evaluation of emotional understanding. Also, the test results are of high reliability, being able to distinguish high temporal stability 
after the month. The results of the correlations were higher than in the validation processes mentioned above. In these processes the time previous to the re-test has been greater.

The information functions of the items conform to the theoretically expected pattern, its hierarchical structure determines that the last items should measure more accurately the high levels of the trait, since they are the ones with the lowest results, as well as the ones with the greatest discriminative power, which could be seen in the item-total correlations. These results are similar to those of other studies conducted for the validation of this test in other populations (Albanese y Molina, 2008; Fidalgo, Pons, Quintanilla, 2012; Roazzi, Dias, Minervino y Pons, 2008), this could explain the non-necessary correlation between the indicators found in this study.

No significant differences were observed according to sex and age, except for some aspects of the reflexive level such as the recognition of mixed emotions or emotional ambivalence, which contrasts with approaches of some authors (Pons et al., 2014). This is explained because the age range was very small, which suggests that the research should be expanded, applied in other ages within the child population.

To conclude, the study allowed to verify the adjustment of the instrument and the feasibility of its application, from a first rapprochement. However, it is recommended to continue advancing in the finding of other types of validity evidence, from other psychometric measures in futures studies with larger and stratified samples. This would allow the realization of cross validation analysis to examine if the same factorial model and the values of the parameters are maintained through sex, age, as well as other variables. The use of the technique in the Cuban population will have a significant impact for children assessment, where it would be interesting to study, not only the particularities of affective development in healthy samples, but it would favor early diagnosis and intervention in different clinical samples. 
